\title{
Coronary artery computed tomography as the first-choice imaging diagnostics in patients with high pre-test probability of coronary artery disease (CAT-CAD)
}

\author{
Piotr N. Rudziński ${ }^{1}$, Mariusz Kruk ${ }^{1}$, Marcin Demkow ${ }^{1}$, Zofia Dzielińska ${ }^{1}$, Jerzy Pręgowski ${ }^{2}$ Adam Witkowski², \\ Witold Rużyłło ${ }^{1,2}$, Cezary Kępka ${ }^{1}$
}

${ }^{1}$ Department of Coronary and Structural Heart Disease, Institute of Cardiology, Warsaw, Poland

${ }^{2}$ Department of Interventional Cardiology and Angiology, Institute of Cardiology, Warsaw, Poland

Postep Kardiol Inter 2015; 11, 4 (42): 281-284

DOI: $10.5114 /$ pwki.2015.55597

\begin{abstract}
Introduction: The primary diagnostic examination performed in patients with a high pre-test probability of coronary artery disease (CAD) is invasive coronary angiography. Currently, approximately $50 \%$ of all invasive coronary angiographies do not end with percutaneous coronary intervention $(\mathrm{PCl}$ ) because of the absence of significant coronary artery lesions. It is desirable to eliminate such situations. There is an alternative, non-invasive method useful for exclusion of significant CAD, which is coronary computed tomography angiography (CCTA).

Aim: We hypothesize that use of CCTA as the first choice method in the diagnosis of patients with high pre-test probability of CAD may reduce the number of invasive coronary angiographies not followed by interventional treatment. Coronary computed tomography angiography also seems not to be connected with additional risks and costs of the diagnosis. Confirmation of these assumptions may impact cardiology guidelines.

Material and methods: One hundred and twenty patients with indications for invasive coronary angiography determined by current ESC guidelines regarding stable CAD are randomized $1: 1$ to classic invasive coronary angiography group and the CCTA group.

Results: All patients included in the study are monitored for the occurrence of possible end points during the diagnostic and therapeutic cycle (from the first imaging examination to either complete revascularization or disqualification from the invasive treatment), or during the follow-up period.

Conclusions: Based on the literature, it appears that the use of modern CT systems in patients with high pre-test probability of CAD, as well as appropriate clinical interpretation of the imaging study by invasive cardiologists, enables precise planning of invasive therapeutic procedures. Our randomized study will provide data to verify these assumptions.
\end{abstract}

Key words: coronary artery disease, coronary angiography, coronary tomographic angiography, multi-detector computed tomography.

\section{Introduction}

The primary diagnostic examination performed in patients with a high pre-test probability of coronary artery disease (CAD) is invasive coronary angiography. Usually, it requires at least a short-term hospitalization and is associated with significant risk of serious complications, such as aortic dissection, bleeding, stroke, heart attack or death. Currently, approximately $50 \%$ of all invasive coronary angiographies do not end with percutaneous coronary intervention $(\mathrm{PCl})$ because of the absence of significant coronary artery lesions. It is desirable to eliminate such situations.
In 2010 Patel et al. [1] stated that CAD can be diagnosed in only $37.6 \%$ of patients undergoing invasive coronary angiography. The Board of Association of Cardiovascular Interventions of the Polish Cardiac Society reported that approximately $45 \%$ of all invasive coronary angiographies (88 993 procedures) carried out in 2011 in Poland did not end with $\mathrm{PCl}$. More recent data from the ACROSS Registry show that significant stenoses are found in $51 \%$ of patients undergoing coronary angiography and that $\mathrm{PCl}$ is performed in only $46.7 \%$ of patients undergoing the invasive examination [2].

\section{Corresponding author:}

Dr. Mariusz Kruk, Department of Coronary and Structural Heart Disease, Institute of Cardiology, 42 Alpejska St, 04-628 Warsaw, Poland, phone: +48 601145 011, e-mail: mariuszkruk2000@yahoo.com

Received: 2.11.2015, accepted: 2.11.2015. 
There is an alternative, non-invasive method useful for exclusion of significant $C A D$, which is coronary computed tomography angiography (CCTA). It is currently recommended for individuals with intermediate CAD pre-test probability (15-50\%). Higher pre-test probability of CAD is currently a contraindication to CCTA. Limitations associated with the use of CCTA are explained by insufficient predictive value of currently used standard hardware, which is a 64-row CT scanner. The latest CT scanners (more than 64 rows) are characterized by spatial resolution of $0.3 \mathrm{~mm}$ (Somatom Definition FLASH, Siemens, Forchheim, Phillips Brilliance ICT), which is very similar to the level of invasive angiography resolution of $0.2 \mathrm{~mm}$.

Similarly to invasive coronary angiography, during CCTA the patient is exposed to iodine contrast and X-rays. The effective dose of radiation and contrast amount in the non-invasive study carried out with the use of a modern CT scanner is often even lower than during the invasive examination, amounting to $5-6 \mathrm{mSv}$ and $50-80 \mathrm{ml}$ of contrast agent.

The dynamic development of CT techniques, increased spatial resolution, reduced artifacts, and reduction of radiation dose and contrast amount, allow more accurate and safer imaging of coronary arteries [3].

The Institute of Cardiology was one of the first centers in the world to show the usefulness of CCTA in planning of coronary interventions $[4,5]$. In cardiac centers developing an extensive program of CT-based CAD diagnosis, a reduced number of uncompleted coronary interventions is noticeable.

\section{Review of ESC guidelines}

Current ESC recommendations concerning stable CAD indicate invasive coronary angiography as the first choice method in patients with a high pre-test probability of CAD.

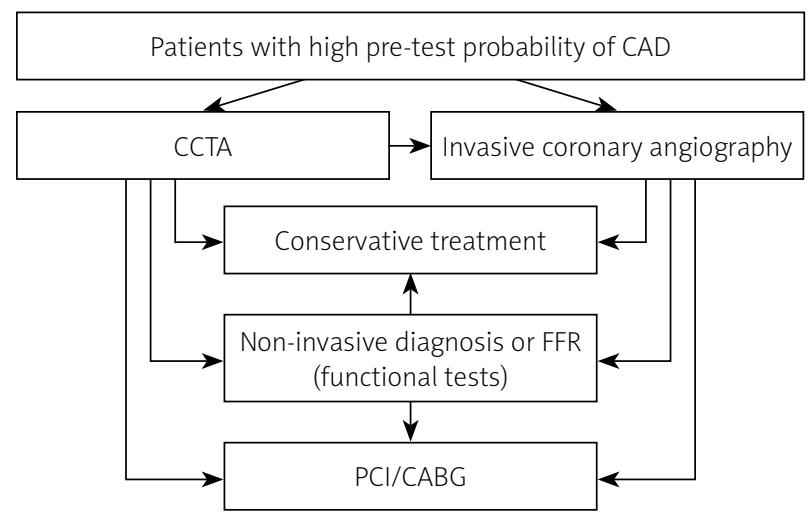

Figure 1. Randomization and further procedures in the CAT-CAD study within the diagnostic and therapeutic cycle (assumed to be complete within 3 months of study entry)

$C A B G$ - Coronary artery bypass graft, $C A D$ - coronary artery disease, CCTA - coronary computed tomography angiography, FFR - fractional flow reserve, $\mathrm{PCl}$ - percutaneous coronary intervention.
It is assumed that patients meeting the above criteria in most cases are candidates for further interventional treatment of CAD. Optimally, invasive investigation should not be undertaken unless further invasive treatment of CAD is not necessary. Nevertheless, as mentioned before, the traditional diagnostic model results in a high proportion of invasive coronary angiographies, which do not result in further therapeutic procedures (around 50\%). The reason for that may be the lack of significant stenoses, the presence of significant stenoses which are not eligible for interventional treatment, or inadequate preparation of the patient for ad-hoc percutaneous treatment.

We hypothesize that use of CCTA as the first choice method in the diagnosis of patients with high pre-test probability of CAD may reduce the number of invasive coronary angiographies not followed by interventional treatment. The CCTA also seems not to be connected with additional risks and costs of the diagnosis. Confirmation of these assumptions may impact cardiology guidelines.

\section{Aim}

This is a prospective, randomized, open-label, single center trial designed to evaluate superiority of CCTA to classic invasive coronary angiography (concerning effectiveness and safety) in the diagnosis of patients with high pre-test probability of stable CAD according to ESC recommendations. Inclusion and exclusion criteria are listed below:

\section{Material and methods}

\section{Inclusion criteria}

1. Age $>18$ years old.

2. Patients providing written informed consent.

3. Indications for elective invasive coronary angiography defined by ESC [6], as:

- Left ventricle ejection fraction < 50\% and typical angina symptoms,

- Probability of CAD due to criteria of age, sex and symptoms $>85 \%$,

- Probability of CAD due to criteria of age, sex and symptoms $50-85 \%$ with positive or intermediate cardiac stress test.

\section{Exclusion criteria}

1. No consent to the study.

2. Acute coronary syndrome.

3. Recurrence of typical angina symptoms 1 year after last PCl.

4. Contraindications to invasive coronary angiography.

5. Glomerular filtration rate (GFR) $<60 \mathrm{ml} / \mathrm{min} / 1.73 \mathrm{~m}^{2}$.

6. Significant arrhythmia.

7. Body mass index $(\mathrm{BMI})>35 \mathrm{~kg} / \mathrm{m}^{2}$.

\section{Methods}

The project is planned to include 120 patients with indications for invasive coronary angiography determined 
by current ESC guidelines regarding stable CAD. Patients are randomized $1: 1$ to $A$. the classic invasive coronary angiography group and B. the CCTA group (Figure 1).

It is assumed that the first invasive procedure in the CCTA group (if necessary) will be performed within 2 weeks of CCTA investigation. Depending on the result of the primary study, patients are qualified to: $A$ - non-invasive treatment, $\mathrm{B}$ - further invasive diagnosis, $\mathrm{C}$ - further non-invasive diagnosis, $D$ - interventional treatment ( $P C I / C A B G)$.

\section{Study end points}

All patients included in the study are monitored for the occurrence of possible end points during the diagnostic and therapeutic cycle (from the first imaging examination to either complete revascularization or disqualification from the invasive treatment), or during the follow-up period.

\section{Primary outcomes}

1. The average number of invasive procedures (coronary angiography/PCI) in the arm: A. CCTA versus B. Classic diagnostics (superiority) during the diagnostic and therapeutic cycle (it is assumed it will be completed within 3 months of the participants' study entry).

2. The proportion of "avoidable" invasive diagnostic procedures (coronary angiographies not followed by an intervention) in the arm: A. CCTA versus B. Classic diagnostics (superiority) during diagnostic and therapeutic cycle.

\section{Safety primary outcomes}

1. The volume of contrast used for diagnosis and possible coronary intervention in the arm: A. CCTA versus B. Classic diagnostics (non-inferiority) during diagnostic and therapeutic cycle (it is assumed it will be completed within 3 months of the participants' study entry).

2. The radiation dose used for diagnosis of CAD and possible intervention in the arm: A. CCTA versus B. Classic diagnostics (non-inferiority) during diagnostic and therapeutic cycle (it is assumed it will be completed within 3 months of the participants' study entry).

\section{Secondary outcomes}

1. Composite outcome - number of serious adverse events during either diagnosis and therapeutic cycle (assumed up to 3 months) or during follow-up (up to 36 months): death, acute coronary syndrome, urgent hospitalization for cardiovascular causes, stroke, unplanned $\mathrm{PCl}$ as a treatment of invasive coronary angiography complications, urgent CABG surgery as a result of $\mathrm{PCl}$ or coronary angiography complications, surgical treatment of local vascular complications or with blood products, hospitalization or prolongation of hospitalization due to local vascular complications, the occurrence of a pseudoaneurysm, fistula, or occlusion in the vascular access site, decrease in renal function (a fall of at least one stage of chronic kidney disease), 2-5 type bleeding defined by the Bleeding Academic Research Consortium, life threat, need for hospitalization or its prolongation, durable or substantial health damage.

2. Time to occurrence of combined end point during either diagnosis and therapeutic cycle (assumed up to 3 months) or during follow-up (up to 36 months): myocardial infarction, death, acute coronary syndrome, unplanned coronary revascularization (including restenosis), urgent hospitalization for cardiovascular reason, stroke.

3. Number of $\mathrm{PCl}$ procedures performed in accordance with ESC recommendations (50\% stenosis + typical symptoms or documented ischemia in the area of vascularization), where the treatment planned on the basis of CCTA is not treated as "ad-hoc" during the diagnostic and therapeutic cycle (assumed up to 3 months).

4. Average number of therapeutic procedures (PCI/CABG) during the diagnostic and therapeutic cycle (assumed up to 3 months).

5. Effectiveness of the diagnostic and therapeutic cycle in $C A D$, defined as: average time to complete the diagnostic and therapeutic cycle.

6. Effectiveness of the diagnostic and therapeutic cycle in $C A D$, defined as: average consumption of resources (comparison of CAD diagnosis and treatment costs in accordance with National Health Service and Institute of Cardiology price lists).

7. Effectiveness of the diagnostic and therapeutic cycle in CAD, defined as: average number of days of hospitalization required to complete the diagnostic and therapeutic cycle.

8. Effectiveness of the diagnostic and therapeutic cycle in $\mathrm{CAD}$, defined as: cost-effectiveness analysis.

\section{Conclusions}

Based on the literature, it appears that the use of modern CT systems in patients with high pre-test probability of CAD, as well as appropriate clinical interpretation of the imaging study by invasive cardiologists, enables precise planning of invasive therapeutic procedures. The proposed diagnostic schema, with the use of CT as the first choice method in CAD diagnosis, may substantially reduce the number of invasive procedures. Moreover, it can enable more precise planning of potential treatment options, increase the comfort and safety of patients, and reduce the time and cost of the diagnostic and therapeutic cycle. Achieving these objectives should be possible by simplifying the diagnostic path and eliminating invasive procedures not followed by intervention. Our randomized study will provide data to verify these assumptions.

Clinical Trials number: NCT02591992.

\section{Conflict of interest}

The authors declare no conflict of interest. 


\section{References}

1. Patel MR, Peterson ED, Dai D, et al. Low diagnostic yield of elective coronary angiography. N Engl J Med 2010; 362: 886-95.

2. Borges Santos M, Ferreira AM, de Araújo Goncalves P, et al. Diagnostic yield of current referral strategies for elective coronary angiography in suspected coronary artery disease-an analysis of the ACROSS registry. Rev Port Cardiol 2013; 32: 483-8.

3. de Araújo Gonçalves P, Jerónimo Sousa P, Calé R, et al. Effective radiation dose of three diagnostic tests in cardiology: single photon emission computed tomography, invasive coronary angiography and cardiac computed tomography angiography. Rev Port Cardiol 2013; 32: 981-6.

4. Pregowski J, Kepka C, Kruk M, et al. Comparison of usefulness of percutaneous coronary intervention guided by angiography plus computed tomography versus angiography alone using intravascular ultrasound end points. Am J Cardiol 2011; 108: 1728-34.

5. Opolski MP, Kepka C, Witkowski A, et al. Coronary computed tomographic angiography for prediction of procedural and intermediate outcome of bypass grafting to left anterior descending artery occlusion with failed visualization on conventional angiography. Am J Cardiol 2012; 109: 1722-8.

6. Task Force Members, Montalescot G, Sechtem U, Achenbach S, et al. 2013 ESC guidelines on the management of stable coronary artery disease: the Task Force on the management of stable coronary artery disease of the European Society of Cardiology. Eur Heart J 2013; 34: 2949-3003. 